\title{
The first optical characterization of the Oukaïmeden site with the Generalized Seeing Monitor (GSM)
}

\author{
A. Ziad ${ }^{1}$, A. Jabiri ${ }^{2}$, Z. Benkhaldoun ${ }^{2}$, F. Martin ${ }^{1}$, R. Conan ${ }^{1}$, M. Lazrek ${ }^{3}$, and J. Borgnino ${ }^{1}$ \\ 1 UMR 6525 Astrophysique, Université de Nice-Sophia Antipolis, Parc Valrose, 06108 Nice Cedex 2, France \\ 2 Laboratoire de Physique des Hautes Énergies et Astrophysique, Département de Physique, \\ Université Cadi Ayyad, Faculté des Sciences Semlalia, BP 2390, Marrakech, Morocco \\ 3 Astronomical and Geophysical Laboratory, C.N.C.P.R.S.T., BP 1346 RP, Rabat, Morocco
}

Received 31 January 2000 / Accepted 17 October 2000

\begin{abstract}
The main atmospheric optical parameters (AOP) have been measured during 10 nights in April 1998 with the GSM instrument at the Moroccan site of Oukaïmeden. These parameters are of interest for the optimization of high angular resolution techniques. During this campaign the temporal evolution of the AOP and their distributions have been studied. The outer scale presents a log-normal histogram with a median value of $31 \mathrm{~m}$ which is rather similar to the values obtained at other sites visited with GSM. The selection of the Oukaïmeden site is the result of several topographical and meteorological studies on the Atlas mountain chain. Since 1988 this site has been chosen for the installation of one of the IRIS (International Research of the Interior of the Sun) stations. Here, we present the whole AOP data measured with GSM during this campaign. The main photometric and meteorological conditions of this site are also presented.
\end{abstract}

Key words. atmospheric effects - turbulence - site testing

\section{Introduction}

Oukaïmeden is a $2700 \mathrm{~m}$ mountain in the Moroccan High Atlas. This site $\left(31^{\circ} 12^{\prime}\right.$ North and $7^{\circ} 52^{\prime}$ West $)$ is located at $70 \mathrm{~km}$ from the city of Marrakesh; it is easily accessible by a fairly good road. The selection of Oukaïmeden as a possible site for the first Moroccan observatory was the result of meteorological, topographical and daytime photometry studies during several years (Kadiri 1983; Benkhaldoun 1994). In 1987, this site was included in the IRIS network (Benkhaldoun et al. 1991) and later tested by the GONG project (Hill 1994a; Hill 1994b). But, since all the previous studies concerned daytime observations and meteorological conditions (Benkhaldoun 1994); nighttime optical characterization of this site was needed in order to develop astronomical programs.

This characterization is related to the effect of atmospheric turbulence on the wavefronts which severely reduces the resolution of ground-based astronomical observations. Different techniques have been developed to achieve the diffraction limited resolution of observing instruments, namely speckle and long baseline interferometry (SI, LBI) and adaptive optics (AO). But the performance of these High Angular Resolution (HAR) methods requires a better understanding of the behavior of

Send offprint requests to: A. Ziad, e-mail: ziad@unice.fr wavefronts perturbed by the atmospheric turbulence, more exactly a better knowledge of atmospheric optical parameters (AOP). Among these parameters are the Fried coherence size $r_{0}$ which is related to the seeing $\epsilon_{0}$ $\left(\epsilon_{0}=0.98 \lambda / r_{0}\right)$, the spatial coherence outer scale $\mathcal{L}_{0}$, the isoplanatic angle $\theta_{0}$ and the wavefront coherence time $\tau_{0}$. It is well known that the performance of an $\mathrm{AO}$ system depends upon the seeing conditions. On the other hand, for passive LBI the outer scale $\mathcal{L}_{0}$ is of interest because its value provides the spectral bandwidth optimizing the signal-to-noise ratio (Rodddier 1981; Ziad et al. 1994a). In addition, the $\mathcal{L}_{0}$ is also a critical parameter for cophasing an interferometer (Mariotti 1993). In the case of a large telescope equipped with $\mathrm{AO}$ system, the residual error after a tip-tilt correction grows for small $\mathcal{L}_{0}$ values (Voitsekhovich \& Cuevas 1995). On the other hand, the choice of the AO reference star must take into account the constraints related to the isoplanatic angle. Finally the knowledge of the wavefront coherence time $\tau_{0}$ is of interest to optimize the exposure time. Indeed, a compromise must be found between the flux, as a function of increasing exposure time, and the degradation of the image quality.

For all these reasons a measurement campaign has been performed at the Oukaïmeden site with the Generalized Seeing Monitor. This instrument, developed at the Département d'Astrophysique of Nice University, 
consists of monitoring all of the optical parameters and especially the outer scale $\mathcal{L}_{0}$ (Martin et al. 1994). This monitor and data reduction procedures are succinctly described in Sect. 3.1. Then, in Sect. 2, we summarize the meteorological conditions of the Oukaïmeden site and the previous daytime characterizations. Finally, in Sect. 4 the summary of the measurement campaign made with GSM at this site is presented and discussed in Sect. 5 .

\section{Oukaïmeden site}

The first step towards the creation of an observatory is the selection of an optimal site. It must be a compromise between the necessary remoteness from any light sources and the proximity to scientific centers. This choice determines the attraction and interest that this future observatory will be able to cause in the international astronomical community.

Based on these various constraints and other topographical and meteorological situations, a detailed study was carried out on the Atlas mountains (Kadiri 1983). The selection of the Oukaïmeden site resulted from the best compromise between all the constraints and appears to be a good choice, when compared to other former observatories, in terms of daytime sky transparency, photometry, extinction and ground climatology. In this section we present a summary of the meteorological conditions and the previous daytime site-testing campaigns which have been carried out at Oukaïmeden site.

\subsection{Previous photometric studies}

As soon as Oukaïmeden was selected as a potential site for a future observatory, daytime measurements were performed at different wavelengths to estimate the diurnal quality of this site.

In 1987, a simple Flux Integration Photometer (FIP) was built to sample different values of atmospheric extinction coefficient. The FIP instrument (Benkhaldoun et al. 1993) is a full disk sunlight triple photometer. The analysis of the FIP database shows that the Oukaïmeden appears to exhibit photometric conditions during $65 \%$ of daytime. The extinction coefficient at $700 \mathrm{~nm}$ wavelength is $k=0.13 \mathrm{mag} /$ air-mass.

The same year brought the installation of an IRIS station at Oukaïmeden. The IRIS instrument (Grec et al. 1991) measures a Doppler shift integrated over the entire solar disk, using the Sodium optical resonance spectrometer. The statistics from one year of IRIS photometric data obtained at Oukaïmeden show that the average extinction coefficient is about $k=0.1 \mathrm{mag} /$ air-mass in the Sodium band and that the daily fraction of clear weather is $64 \%$ (Benkhaldoun \& Siher 1998).

In 1988, the site was also chosen to be tested by the GONG project site survey controlled by the National Solar Observatory of Tucson in Arizona. Thus, the GONG network installed on the site a pyrheliometer to achieve a complementary photometric study of the sunlight.
The results obtained (Hill 1994b) presented a lower value for the clear time fraction (50\%) and an average extinction coefficient of $k=0.12 \mathrm{mag} /$ air-mass. This value is similar to that measured by Benkhaldoun et al. (1993) with the FIP instrument.

The values of the daytime extinction coefficients are satisfactorily consistent. Their small differences depend on details of the instrument calibrations, and also on the different spectral bandwidths used by the different instruments.

The significantly smaller fraction of clear time measured by the GONG site survey instrument has simple explanations depending on data analysis (Hill 1994a; Hill 1994b) as explained by Jabiri et al. (2000). On the other hand, the results obtained with the IRIS and the FIP instruments are close, respectively, $64 \%$ and $65 \%$ of clear daytime. For night-time conditions further measurements with the GSM are needed for a long period (one year) to provide more statistics on the clear time and to show the variations with the seasons.

\subsection{Meteorological conditions}

In 1990 a meteorological station was installed to collect information at the ground of the insolation, the pluviometry, the wind speed and direction and the temperature. The analysis of these meteorological data tends to confirm the choice of this site (Jabiri et al. 2000). The temperature changes weakly during the night and the variation does not exceed 2 or $3{ }^{\circ} \mathrm{C}$. We can thus expect to have weak turbulence close to the ground.

Humidity undergoes a significant variation during the night (Jabiri et al. 2000). It decreases until midnight and starts to stabilize around the nightly average value (40\%). This variation of humidity is checked by daytime photometric measurements which show a transparent sky in the morning, which covers with clouds in the afternoon and starts to get clear at the beginning of evening. The probability of a high local humidity is weak and for night-time conditions this humidity is lower than $60 \%$ (Jabiri et al. 2000).

During the night (Fig. 1), generally the wind is blowing from the south and dispersed from east to west. This wind blows predominantly from SE and most of the time it is lower than $5 \mathrm{~m} / \mathrm{s}$. This wind is responsible for Sahara dust transport; but the presence of high mountains towards the East of the Oukaïmeden protects the site from this dust wind.

\section{The GSM campaign at the Oukaïmeden site}

\subsection{The Generalized Seeing Instrument}

The GSM instrument consists of evaluating the optical parameters of the perturbed wavefront by measuring Angle of Arrival (AA) fluctuations. Indeed, the GSM uses the same principle than a Shack-Hartmann, i.e., measuring $\mathrm{AA}$ at different points of the wavefront and computing 


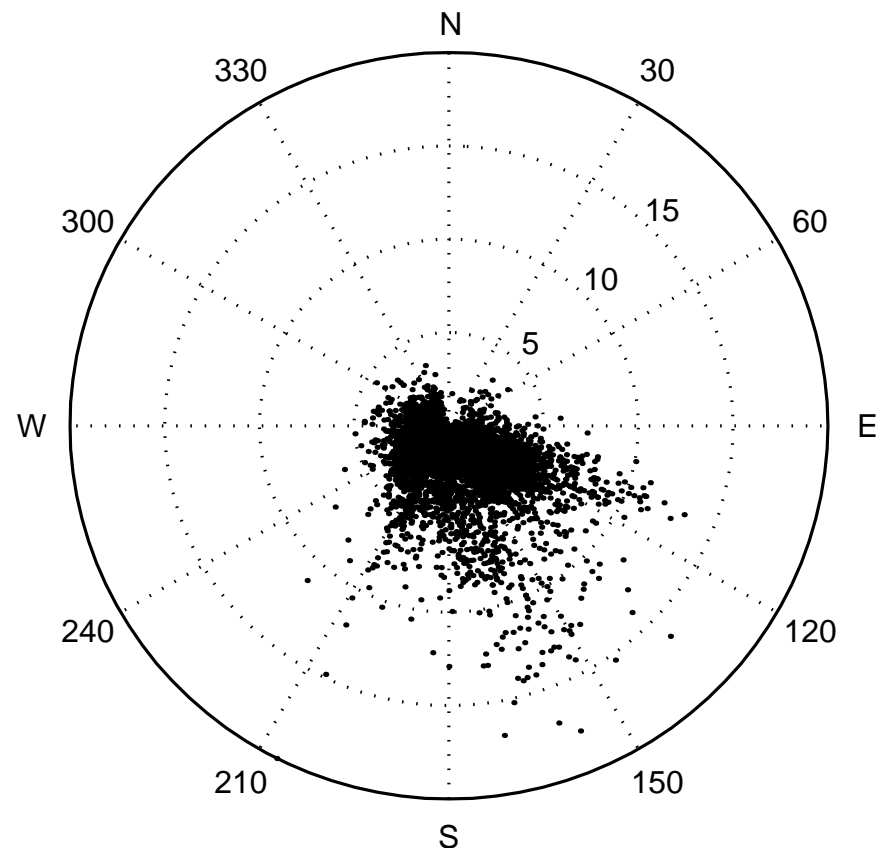

Fig. 1. Night-time windrose versus modulus at the Oukaïmeden site. These data are the results of more than 2 year measurements (December 1990 to March 1993)

AA spatio-temporal correlations leads to estimates of the seeing $\epsilon_{0}$, outer scale $\mathcal{L}_{0}$, isoplanatic angle $\theta_{0}$ and coherence time $\tau_{0}$.

The instrument consists of four 10-cm telescopes on equatorial mounts (Fig. 2) equipped with detection modules measuring the AA fluctuations and interfaced to a computer PC managing simultaneously the 4 modules. Each telescope, pointing at the same star, measures the AA fluctuations by means of flux modulation which is produced by the displacement of the star image over a Ronchi grating. Two telescopes are installed on a common mount on a central pier (Fig. 2) working as a differential image motion monitor (DIMM) with a $25 \mathrm{~cm}$ baseline. Two other telescopes have different mounts on separate piers, located $0.8 \mathrm{~m}$ to the south and $1 \mathrm{~m}$ to the east from the central pier, thus forming an L-shaped configuration. This configuration has been chosen for more sensitivity to the outer scale. The telescopes were situated $1.7 \mathrm{~m}$ above the ground.

The AA fluctuations are measured with $5 \mathrm{~ms}$ resolution time during 2 min acquisition time. Data are processed immediately after each acquisition, allowing a quasi real-time monitoring of the AOP. The data acquisition is repeated typically every $4 \mathrm{~min}$.

The AA covariances are computed for each baseline (6 baselines with 4 GSM modules) and normalized by the differential variance of AA on the $25-\mathrm{cm}$ baseline. They are compared to Von Kàrmàn theoretical normalized covariances (Avila 1997) and the appropriate $\mathcal{L}_{0}$ is found for each baseline. The final value of $\mathcal{L}_{0}$ is taken as the median of the 6 individual $\mathcal{L}_{0}$ values and its error is

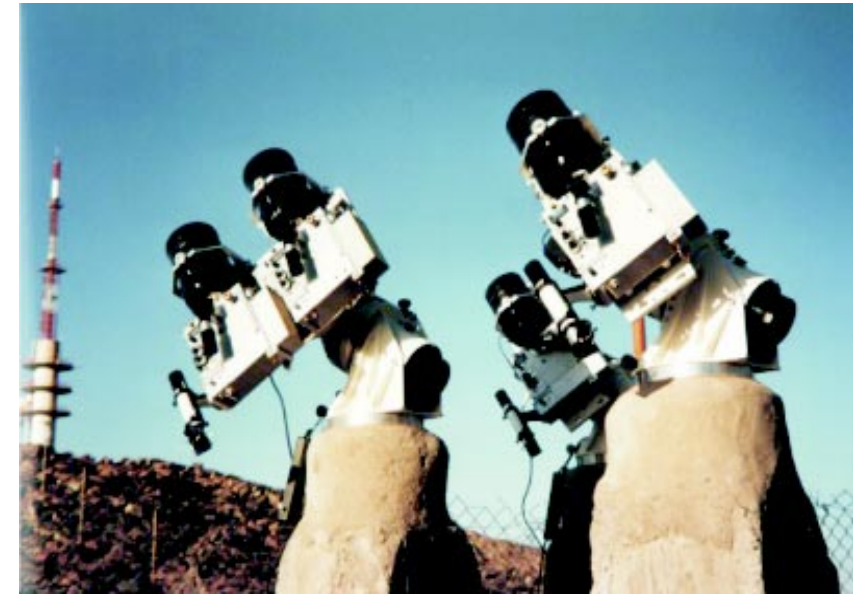

Fig. 2. GSM at the Oukaïmeden site

estimated. The seeing $\epsilon_{0}$ is calculated from the differential variance given by the coupled modules as in the DIMM instrument (Sarazin \& Roddier 1990). The scintillation index $\sigma_{\mathrm{I}}^{2}$ is computed during data reduction and, as suggested by Loos \& Hogge (1979) and Krause-Polstorff et al. (1993), an estimate of the isoplanatic angle is deduced.

A quantification of the different GSM noises has been performed and hence corrections of photon and scintillation noises are done before data processing. Another correction for finite exposure time is also performed; it consists in computing AA variance (or covariance) for $5 \mathrm{~ms}$ and $10 \mathrm{~ms}$ and in extrapolating linearly to the $0 \mathrm{~ms}$ exposure time. Finally, the statistical errors of the computed variances and covariances are estimated and consequently the errors of the AOP measured with GSM are provided.

In order to check the wind shake effect, $r_{0}$ is computed from absolute image motion in each telescope, corrected for finite $\mathcal{L}_{0}$ (Ziad et al. 1994b) and compared to $r_{0}$ provided by the differential technique. A good agreement is found for ground wind speed less than $10 \mathrm{~m} / \mathrm{s}$, showing that telescope vibrations were not significant.

\subsection{GSM configuration at the site}

The on-site mission preparation consisted in building 4 piers to support the GSM different modules. The IRIS building had been arranged before our arrival to serve as GSM office. It was used before observations to store the GSM equipment.

The GSM was installed on three piers located near the IRIS instrument on a pre-existing platform. The piers had a compact L-shaped configuration as described in Sect. 3.1.

The wind-induced telescope vibrations present a potential problem in AA measurements. To reduce this effect, the instrument was surrounded by a protective net (30\% wind transparency) from 3 sides (south, east and west). The net was $2 \mathrm{~m}$ high, and completely decoupled from piers. 


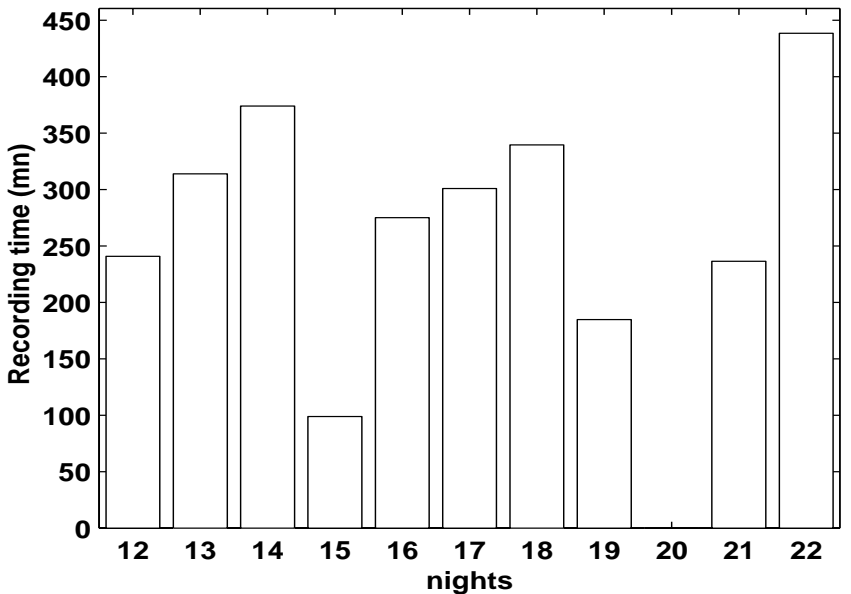

Fig. 3. The distribution of the total measuring time during 10 nights at the Oukaïmeden site

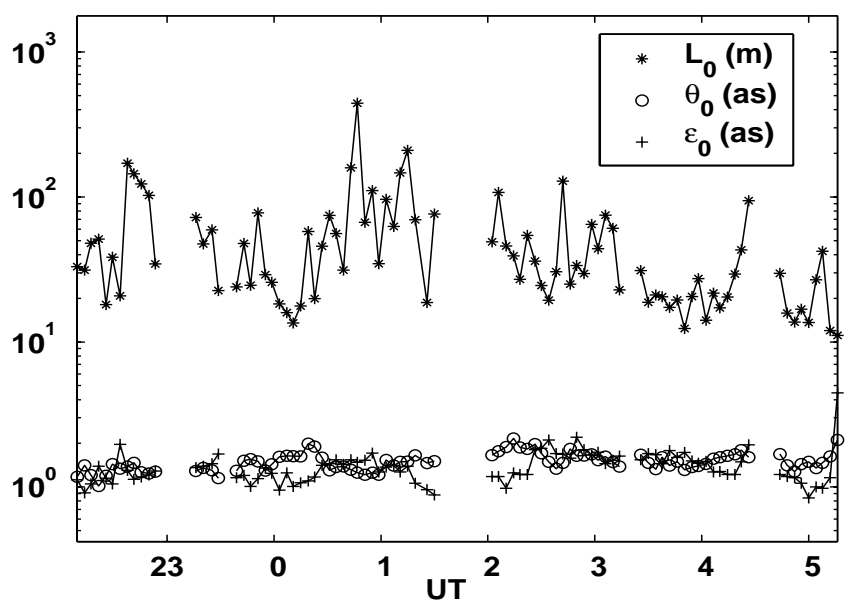

Fig. 4. The AOP measurements during one night (04/14/98) at the Oukaïmeden site

The IRIS building with computers and observers was located at a distance around $4 \mathrm{~m}$ from the central pier, to the south-west. With a prevailing wind from the SE the GSM was supposed to be free from the locally generated turbulence, and the building was not troublesome either. On the other hand, with the southern-west wind the GSM was in the turbulent lee created by its own building and by the small rocks to the south.

A stellar source was selected from the list of single bright stars having from 2 to 3 magnitude and passing close to zenith at the Oukaïmeden site during this season. Due to the limitation of the possible hour angles (in the adopted configuration the telescopes could not be pointed at hour angles less than $1 \mathrm{~h}$ before meridian because they touched the piers) the selected star was usually some $30-40 \mathrm{~min}$. before meridian at the start, and $2-3 \mathrm{~h}$ after meridian at the end of its observations (depending on the availability of a more suitable source). Thus, observations were obtained at zenith angles from $0^{\circ}$ to $45^{\circ}$.

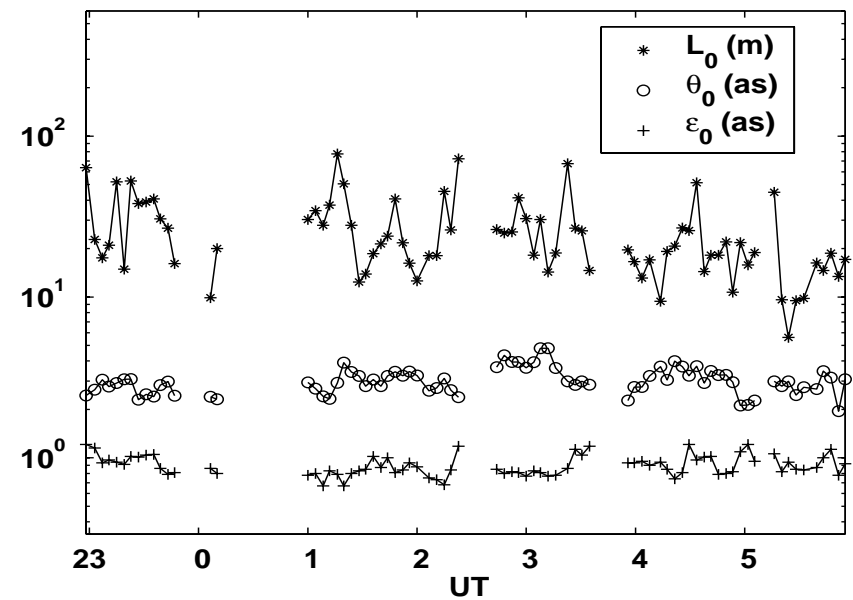

Fig. 5. The AOP measurements during the best night $(04 / 18 / 98)$ at the Oukaïmeden site. The median value of the seeing is 0.85 arcsec

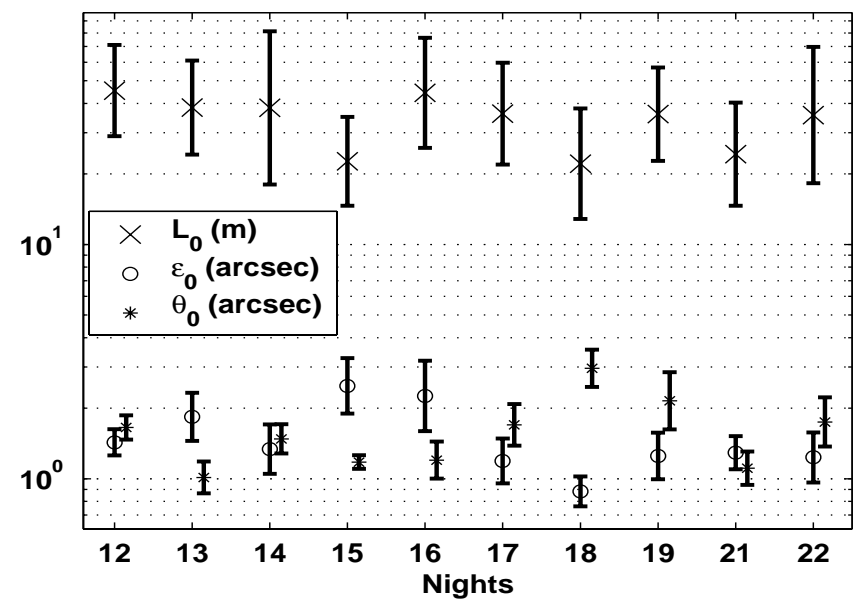

Fig. 6. The summary of AOP data measured during 10 nights at the Oukaïmeden site. The reported values correspond to the AOP logarithmic means and bars indicate intervals containing $68 \%$ of parameter values for each night (mean $\pm 1 \sigma$ of the log-normal nightly distributions)

After pointing to the source, the telescopes were focused by maximizing the modulation contrast. Then, the acquisition sequence was started, interrupted every 30 $40 \mathrm{~min}$ for re-centering of the star in the field of view. Signal was normally recorded during $2 \mathrm{mn}$, and these acquisitions were repeated every $4 \mathrm{mn}$. Occasionally, longer or shorter acquisition times were used for exploratory purpose. Immediately after acquisition the data were transferred to the hard disk and processed. This enabled assessment of data quality and results, including the seeing $\epsilon_{0}$, outer scale $\mathcal{L}_{0}$ and isoplanatic angle $\theta_{0}$.

\section{Results of atmospheric optical parameters with GSM}

During this campaign continuous measurements of the $\operatorname{AOP}\left(\epsilon_{0}, \mathcal{L}_{0}, \theta_{0}\right)$ were obtained during 10 nights in the 

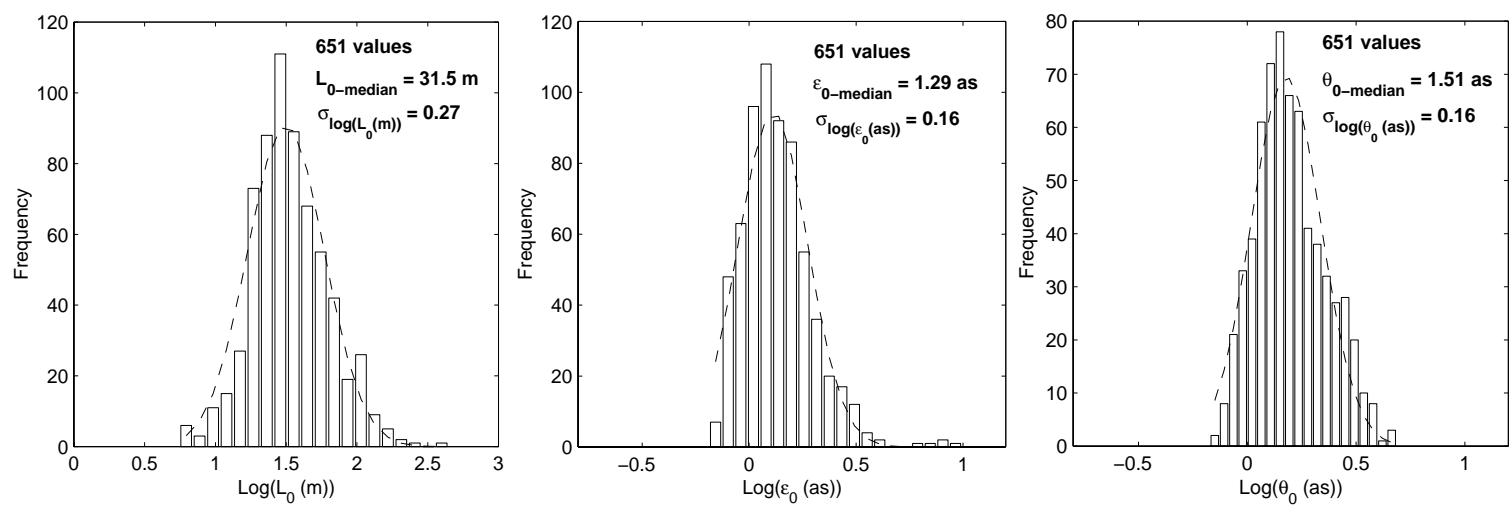

Fig. 7. Histograms of the AOP measured with GSM during 10 nights at the Oukaïmeden site in April 1998. All these parameters are well fitted with log-normal distributions (dashed-line)

period of 10-25 April 1998. These parameters were estimated for the wavelength $\lambda=0.5 \mu \mathrm{m}$. Figure 3 shows the distribution of the total measuring time during this campaign. We draw attention to the larger number of measurements of the last night compared to the others. This data recording time depends on the meteorological conditions (cloud passages, strong wind...), on the observed star change and on the instrument technical problems. Despite this, for this campaign the mean measuring time was more than 4 hours per night providing a high number of measurements of the seeing, outer scale and isoplanatic angle.

A typical temporal evolution of the AOP during one night is shown in Fig. 4. A rapid variation of $\mathcal{L}_{0}$ is usually observed, as well as isolated "bursts" of large outer scale values. These bursts have been also noted at the other sites visited by the GSM. They are typical of our data set, being more frequent on some nights, less frequent or absent on some other nights. Burst duration is about few minutes. Figure 5 shows the best night measurements during this campaign. One can remark that the seeing is usually under 1 arcsec and that all the AOP are less dispersed than the results shown in Fig. 4 which indicates the stability of the conditions during this night. The median value of the seeing is 0.85 arcsec and the best measured value is 0.66 arcsec.

Figure 6 presents the summary of the AOP measurements during this campaign. The seeing, outer scale and isoplanatic angle are well fitted with log-normal distributions (Fig. 7). So, for each night the reported AOP values in Fig. 6 are deduced from the nightly log-normal distributions (mean $\pm 1 \sigma$ ). One can remark that contrary to one night measurements (Fig. 4) all of the AOP have a slow temporal variability from one night to another.

No significant correlation exists between outer scale and seeing but for some nights, as illustrated in Figs. 4 and 6 , there is a correlation between seeing and isoplanatic angle which is due to the contribution of high turbulent layers on the seeing degradation.

\section{Discussion and conclusion}

For the first time the seeing $\epsilon_{0}$, the outer scale $\mathcal{L}_{0}$ and the isoplanatic angle $\theta_{0}$ were monitored continuously during 10 nights at the Oukaïmeden site.

The outer scale $\mathcal{L}_{0}$ data provided by GSM shows that this parameter is well fitted by a log-normal distribution with a rather similar median value compared to the other sites visited by the GSM (Ziad et al. 2000). On the other hand, the $\mathcal{L}_{0}$ measurements during one night (Fig. 4) present a strong temporal variability with isolated "bursts" which suggests a parallel $\mathcal{L}_{0}$ monitoring with HAR observations. The seeing data present some excellent nights with values comparable to La Silla observatory (Fig. 5) but during some other nights the seeing was worse. For this parameter further measurements are needed for a long period (one year) to provide more statistics and to show the variations with the seasons. For the isoplanatic angle $\theta_{0}$, the data are well fitted with a lognormal distribution having a median value of 1.32 arcsec which is comparable to those found at La Silla and Paranal observatories (Ziad et al. 2000). As shown in Fig. 5, during some nights this site presents conditions comparable to the best sites in the world but it would be interesting to know how often this case is produced. No significant correlation exists between the $\mathcal{L}_{0}$ and the seeing $\epsilon_{0}$ but there is one between $\epsilon_{0}$ and the isoplanatic angle $\theta_{0}$ due to the high turbulent layer contribution.

The main meteorological results of this site obtained during more than 2 years are characterized by a SE predominant wind generally lower than $5 \mathrm{~m} / \mathrm{s}$. This site is protected from the dusts coming from Sahara by the Atlas mountains. During the night weak temperature variations are observed that do not exceed $2^{\circ}$ or $3^{\circ}$ showing the relative stability of this site. The humidity is under $60 \%$ in the $100 \%$ of the nightly time.

On the other hand, the FIP, IRIS and GONG data show that the extinction coefficient varies from 0.08 to $0.2 \mathrm{mag} /$ air-mass, and that $64 \%$ (FIP and IRIS) of the time is clear, showing that this site has good photometric conditions. 
Acknowledgements. This campaign has been organized in the framework of the Franco-Moroccan cooperation. The authors would like to thank the Rectorat de l'Université Cadi Ayyad and the Faculté Semlalia for the efficient support of this campaign, the Royal Air Maroc who helped us for financial support of the transport of the GSM instrument. We express our thanks to Eric FOSSAT for his suggestions to improve this manuscript.

\section{References}

Avila, R., Ziad, A., Borgnino, J., et al. 1997, J. Opt. Soc. Am., 14(11), 3070

Benkhaldoun, Z. 1994, Ph.D. Thesis, Université Cadi Ayyad

Benkhaldoun, Z., Kadiri, S., Lazrek, M., \& Touma, H. 1991, Solar Phys., 133, 61

Benkhaldoun, Z., Kadiri, S., Lazrek, M., \& Vernin, J. 1993, Exper. Astron., 2, 345

Benkhaldoun, Z., \& Siher, E. 1-4 June 98, Proceeding of the Soho/Gong98 workshop, Boston (USA), 109-113

Grec, G., Fossat, E., Gelly, B., \& Schmider, F. 1991, Solar Phys., 133, 13
Hill, F. 1994, Solar Phys., 152, 321

Hill, F. 1994, Solar Phys., 152, 351

Jabiri, A., Benkhaldoun, Z., Vernin, J., \& Muñoz-Tuñon, C. 2000, submitted to Astron. Astrophys.

Kadiri, S. 1983, Ph.D. Thesis, Université de Nice

Krause-Polstorff, J., Edmund, A., \& Donald, L. W. 1993, Appl. Opt., 32(21), 4051

Loos, G., \& Hogge, C. 1979, Appl. Opt., 18(15), 2654

Mariotti, J. 1993, Adaptive Optics for Astronomy, ed. D. Alloin, \& J. M. Mariotti (Kluwer Academic Publishers), 309-320

Martin, F., Tokovinin, A., Agabi, A., Borgnino, J., \& Ziad, A. 1994, A\&AS, 108, 173

Roddier, F. 1981, in Progr. Opt., vol. XIX, ed. E. Wolf

Sarazin, M., \& Roddier, F. 1990, A\&A, 227, 294

Voitsekhovich, V., \& Cuevas, S. 1995, J. Opt. Soc. Am., 12(11), 2523

Ziad, A., Borgnino, J., Agabi, A., \& Martin, F. 1994a, Exp. Astron., 5, 247

Ziad, A., Borgnino, J., Martin, F., \& Agabi, A. 1994b, A\&A, 282, 1021

Ziad, A., Conan, R., Tokovinin, A., Martin, F., \& Borgnino, J. 2000, Appl. Opt., in press 